\title{
SOME COVERING PROPERTIES OF LOCALLY UNIVALENT FUNCTIONS
}

\author{
D.A. Brannan and A.K. Lyzzaik
}

\section{Introduction}

The whole question of the covering properties of holomorphic functions has been seminal in complex analysis. In this paper we study some questions that have received surprisingly little attention until recently, concerning the covering properties of the functions holomorphic and locally univalent in the unit disc $\mathbf{B}=$ $\{z:|z|<1\}$.

Liouville's theorem shows that it is not possible for a function $f$ holomorphic and univalent in $\mathbf{B}$ to 'cover' the whole complex plane $\mathbf{C}$ (that is, $f(\mathbf{B}) \neq \mathbf{C}$ ); indeed $\mathbf{C} \backslash f(\mathbf{B})$ must contain an unbounded continuum $\Gamma$. The study of $\mathbf{C} \backslash f(\mathbf{B})$ is a major area of univalent function theory.

The investigation into analogous results for locally-univalent functions $f$ holomorphic in $\mathbf{B}$, with very precise restrictions on the number of times points of $\mathbf{C}$ may be covered by the Riemann surface $f(\mathbf{B})$, may be said to have been initiated by [2]. There it was shown that if $f$ is holomorphic, locally-univalent and $p$-valent in $\mathbf{B}$, then $v(f) \neq\{p-1, p\}$, where $v(f)=\left\{\operatorname{card} f^{-1}(w): w \in \mathbf{C}\right\}$. (In particular, there exists some point $w_{0}$ that is covered by $f$ at most $(p-2)$ times; for example, if $p=2$ then $f(\mathbf{B}) \neq \mathbf{C}$.)

The idea of the deficiency of a $p$-valent holomorphic function in $\mathbf{B}$ was introduced in [2]; the deficiency of $f$ at $w, \delta(w)$, is defined as

$$
\delta(w)=\max \left\{0, p-1-\operatorname{card} f^{-1}(w)\right\}
$$

where card $f^{-1}(w)$ takes account of multiplicity, and the deficiency of $f$ is the sum $\sum_{w \in \mathbf{C}} \delta(w)$. Answering conjectures in [2], A. Lyzzaik and D. Styer [6] proved that (A) a locally-univalent, $p$-valent holomorphic function in $\mathbf{B}$ satisfies the inequality $\delta(f) \geq p-1$, and (B) a $p$-valent holomorphic function in $\mathbf{B}$ with $\delta(f)=0$ must have at least $(p-1)$ critical points. Their technique was to embed the images of such functions in $p$-sheeted covering surfaces over the Riemann sphere $\mathbf{P}$.

Some of the work in [2] and [6] was generalized in [14] to functions between $n$-dimensional manifolds, in [15] to ramified meromorphic functions in arbitrary domains, in [16] to analytic functions between Riemann surfaces, and in [17] to finite-to-one open continuous functions between surfaces. 
Recently, M. Ortel and W. Smith [8] have shown that if $f$ is holomorphic, locally-univalent and $p$-valent in $\mathbf{B}$, then $v(f) \neq\{1, p\}$. They also conjectured that for every $q$ with $1<q<p$, then $v(f) \neq\{q, p\}$; a main result in this paper is a proof of a generalization of that conjecture.

\subsection{Two covering surface theorems}

We will say that a function $f$ satisfies the hypotheses $(p, q)$ if $f$ is holomorphic and locally univalent in $\mathbf{B}, p$ and $q$ are positive integers with $p>q$, and $f$ covers every point in the image plane either $p$ times or $q$ times, with at most finitely many exceptions. We will call each of these exceptions an exceptional point; it turns out (Lemma 2.4) that exceptional points can be covered at most $(q-1)$ times.

The first main result in this paper is as follows:

Theorem 1. Let the function $f$ satisfy the hypotheses $(p, q)$ and let $e_{1}, \ldots, e_{r}$ be the corresponding exceptional points. Then the Riemann surface $Y$ of the image of $f$ can be embedded in a compact, analytic, $p$-sheeted covering surface $X$ of $\mathbf{P}$. $X$ has branch points over some of the exceptional points, and possibly also over infinity.

In our work it will be valuable to have the following notation available.

Definition 1.1. By the letters $P, Q$ we will denote the sets of all points in the plane that are covered by $f$ exactly $p, q$ times, respectively, and by $E$ the set of exceptional points $e_{1}, \ldots, e_{r}$ (as defined above).

The proof of Theorem 1 follows from a number of lemmas, two of which (Lemmas 2.7 and 3.4) play a central role. The first shows that $P$ is open, and that $f^{-1}(P)$ is a disjoint union of exactly $p$ 'copies' of $P$; the second shows that there is an open neighbourhood $V$ of $Q$ such that $f^{-1}(V)$ contains exactly $q$ copies of $V$.

The key ideas in the rest of the proof are then essentially the same as those in the main theorem of [6]. Over $V$ we tack on $p-q$ new copies of $V$. Then we adjoin these copies to the surface $Y$ by pasting each copy to one and only one of the " $(p-q)$ th" copies of the components of $P \cap V$, and compactify the surface thus obtained by filling up the finite number of points over $E$ and infinity.

The second main result of the paper offers further useful information about the covering properties of the surface $X$ of Theorem 1, provided that $f$ satisfies the additional condition that $E \cup\{\infty\}$ is arcwise connected in $Q \cup E \cup\{\infty\}$. This condition is somehow reasonable in view of Lemma 2.6 in which we show that $Q \cup E \cup\{\infty\}$ is connected. To state our result, we extend the deficiency notion for functions satisfying the hypotheses $(p, q)$ as follows. The $q$-deficiency of $f$ at $w$ is then defined to be

$$
\delta_{q}(w)=\max \left\{0, q-\operatorname{card} f^{-1}(w)\right\},
$$


where card $f^{-1}(w)$ takes multiplicity into account, and the $q$-deficiency of $f$, $\delta_{q}(f)$, is the sum $\delta_{q}(f)=\sum_{w \in \mathbf{C}} \delta_{q}(w)$.

Theorem 2. Under the assumptions of Theorem 1, let the points of the set $E \cup\{\infty\}$ be arcwise connected in the set $Q \cup E \cup\{\infty\}$. Then the surface $X$ of Theorem 1 can be chosen so that the sum of the orders of the branch points over each exceptional point $e_{i}$ is at most $\delta_{q}\left(e_{i}\right)$.

In Section 6 we look at consequences of Theorems 1 and 2 relating the cardinality, $r$, of $E$ and the deficiency $\delta_{q}(f)$, finding in particular that

$$
r(p-q-1)+\delta_{q}(f) \geq p-1
$$

for all functions satisfying the hypotheses $(p, q)$; and that $\delta_{q}(f) \geq p-1$, provided that all the functions satisfy an additional condition (see Theorem 4 in Section 6).

In Section 7 we meet analogous results where analyticity is replaced by continuity and local injectivity. In particular we prove that there is no continuous locally one-one function $f: \mathbf{B} \rightarrow \mathbf{C}$ whose set of valencies is a doubleton $\{p, q\}$, where $p>q \geq 1$; this proves a conjecture of Ortel and Smith [8]. We also consider analogous problems for functions mapping between domains whose connectivity may be positive.

Finally in Section 8 , in a slightly different direction, we present partial results on the problem of identifying in general those subsets of the non-negative integers that can occur as the set $v(f)$ for some function $f$ analytic and univalent in $\mathbf{B}$.

\section{Some topological considerations}

In this section we investigate some of those topological properties of the sets $P, Q$ and $E$ (as defined in Definition 1.1.) that will be needed later.

A key tool in our work will be

Lemma 2.1. Let $f$ be a holomorphic locally-univalent function in $\mathbf{B}$, and $\tilde{\gamma}$ and $\gamma$ simple closed curves in $\mathbf{B}$ and $\mathbf{C}$, respectively, such that $f(\tilde{\gamma})=\gamma$. Then, if $\tilde{G}$ and $G$ are the inner domains of $\tilde{\gamma}$ and $\gamma$, respectively, $f$ is univalent in $\tilde{G} \cup \tilde{\gamma}$ and $f(\tilde{G})=G$.

This is very similar to results for $\tilde{\gamma}=\{|z|=r\}$ in [18; p. 201], [9; Problem 188, p. 141], [10] and [3; pp. 197 and 198].

Proof. First, we have $\partial f(\tilde{G}) \subset f(\partial \tilde{G})=f(\tilde{\gamma})=\gamma$ since $f$ is open. Therefore $f(\tilde{G})=G$ by the Jordan Separation Theorem. Now $f(\partial \tilde{G})=f(\tilde{\gamma})=\gamma=\partial f(\tilde{G})$ implies $\left.f\right|_{\tilde{G}}$ is a covering map. Finally $G$ is simply connected, hence by the monodromy theorem $\left.f\right|_{\tilde{G}}$ is univalent.

We will make much use of the following: 
Lemma 2.2. Let the function $f$ satisfy the hypotheses $(p, q)$ of Section 1; let $z_{0} \in \mathbf{B}$ and $w_{0}=f\left(z_{0}\right)$. Then for any arc $\gamma$, with initial point $w_{0}$, that lies completely either in the set $P$ or in the set $Q$, there exists a unique arc $\tilde{\gamma}$, with initial point $z_{0}$, such that $f(\tilde{\gamma})=\gamma$.

Proof. If a continuation of a branch of $f^{-1}$ along $\gamma$ ends at a point $w_{1}$ which is inner in $\gamma$, then the lift of $\gamma$ tends to $\partial \mathbf{B}$ and hence $w_{1}$ is a cluster point of $f$. Therefore, points on $\gamma$ which are sufficiently close to $w_{1}$ are covered at least $q+1$ times ( $q$ times by a neighbourhood of $f^{-1}\left(w_{1}\right)$ and at least once by points near $\partial \mathbf{B})$, contradicting the assumptions on $\gamma$.

Next we use Lemma 2.2 to study the nature of the set $P$, described in Definition 1.1, associated with a function $f$ satisfying the hypotheses $(p, q)$.

Lemma 2.3. The set $P$ is open, and each of its connected components is a simply-connected domain in $\mathbf{C}_{w}$.

Proof. It is obvious that $P$ is open.

Next, let $P_{1}$ be any connected component of $P$; since $P$ (and so $P_{1}$ ) is open, $P_{1}$ must be a domain. Let $\gamma$ be any simple closed curve in $P_{1}$; we have to show that $\gamma$ is homotopic in $P_{1}$ to a point.

Since $f$ is locally univalent, it follows from Lemma 2.2 that $f^{-1}(\gamma)$ is the union of exactly $p$ simple closed curves $\tilde{\gamma}_{j}, 1 \leq j \leq p$, such that if $\tilde{G}_{j}$ is the inner domain of $\tilde{\gamma}_{j}$, then the domains $\tilde{G}_{j}$ are disjoint, $f$ is univalent on each set $\tilde{G}_{j} \cup \tilde{\gamma}=\tilde{G}_{j}$, and each image domain $f\left(\tilde{G}_{j}\right)$ is the inner domain of $\gamma$.

It follows that every point in the inner domain of $\gamma$ is covered exactly $p$ times, and consequently this domain is contained in $P_{1}$. Therefore $\gamma$ is homotopic in $P_{1}$ to a point, and the proof is complete.

As an application of this result we can deduce an analogous result concerning the covering of the set $E$ of Definition 1.1.

Lemma 2.4. Each point of the set $E$ is covered by $f$ at most $(q-1)$ times.

Proof. Suppose, on the contrary, that there exists some point $e \in E$ that is covered at least $q$ times - and so at least $(q+1)$ times, since $e \notin Q$. It follows from the open mapping theorem that there exists some disc centred at $e$ such that every point in the punctured disc is covered at least $(q+1)$ times; the disc can clearly be chosen sufficiently small that its closure contains no exceptional point other than $e$. The finiteness of $E$ and the restriction on valencies then show that every point in the punctured disc must be covered exactly $p$ times, so that every point of the punctured disc lies in some connected component of $P$. The existence of the 'isolated' point $e$ then contradicts the simple-connectivity of the connected components of $P$ (Lemma 2.3); this contradiction completes the proof.

Our next two results give some information about the location in $\mathbf{C}$ of the set $E$ vis-à-vis $P$ and $Q$. 
Lemma 2.5. The set $E$ is a subset of $\partial P \cap \partial Q$.

Proof. Suppose, on the contrary, that there exists some point $e \in E$ that is not a common boundary point of $P$ and $Q$.

Since $\mathbf{C}=P \cup Q \cup E$ where $P, Q$ and $E$ are disjoint and $E$ is a finite set, it follows that necessarily either $e \in \partial P$ or $e \in \partial Q$.

Suppose, firstly, that $e \in \partial P$. Since $e \notin \partial Q$, the above decomposition of the plane shows that there exists an open disc $D$ centred at $e$ such that all the points of $D \backslash\{e\}$ lie in $P$; this is impossible, by Lemma 2.3.

Suppose, secondly, that $e \in \partial Q$. Just as before it follows that there exists some open disc $D$ centred at $e$ such that all the points of $D \backslash\{e\}$ lie in $Q$. Let $\gamma$ be a circle centred at $e$ that lies in $D$. As in the proof of Lemma 2.3, Lemmas 2.1 and 2.2 imply that $f^{-1}(\gamma)$ is the union of exactly $q$ mutually disjoint simple closed curves in B. Moreover, the inner domains of the curves are also mutually disjoint, and each is mapped homeomorphically under $f$ to the inner domain of $\gamma$. It follows that the exceptional point $e$ must be covered by $f$ at least $q$ times, which is impossible by Lemma 2.4 .

It follows that $e \in \partial P \cap \partial Q$.

Lemma 2.6. The set $Q \cup E \cup\{\infty\}$ is a continuum on the Riemann sphere, and every connected component of $Q \cup E$ is unbounded in $\mathbf{C}$.

Proof. The first statement follows from Lemma 2.3 and Theorems 10.2 and 10.3 in [7, p. 154]. The second statement follows at once, since the Riemann sphere is compact.

Before stating our next result, it is helpful to introduce some convenient terminology.

Definition 2.1. Let $X, Y$ be two topological spaces, and let $G, U$ be open subsets of $X, Y$, respectively. Then if a map $f: X \rightarrow Y$ maps $G$ homeomorphically onto $U, G$ is said to be a copy of $U$ under $f$.

For convenience we will often use the notation $\tilde{U}$ to denote a (particular) copy of an open set $U$ under a function $f$ (such as a function $f$ satisfying hypotheses $(p, q))$.

The next result plays a crucial role in our work.

Lemma 2.7. Let the function $f$ satisfy the hypotheses $(p, q)$ of Section 1 . Then the set $f^{-1}(P)$ consists of exactly $p$ mutually disjoint copies of $P$.

Proof. Let $P_{1}$ be any connected component of $P$; since $P$ is open, so is $P_{1}$. Then clearly it is sufficient to prove the result for $P_{1}$ in place of $P$.

Let $w$ be an arbitrary point in $P_{1}$, and let $\gamma$ be an arc in $P_{1}$ with initial point $w$. By virtue of Lemma 2.2 every branch of $f^{-1}$ that satisfies the normalization $f^{-1}(w)=z_{j}$ for some $j, 1 \leq j \leq p$, can be continued analytically via $f^{-1}$ along $\gamma$. By arguing in essentially the same manner as in Lemma 2.1 the desired result holds. This completes the proof. 


\section{The copies of a neighbourhood of $Q$}

In this section we continue our discussion of functions satisfying the hypotheses $(p, q)$.

We will construct, in several steps, a particular open neighbourhood of the set $Q$ whose pre-image under $f$ contains $q$ mutually disjoint copies of that neighbourhood.

\subsection{A covering of $Q$}

For each point in $Q$ there exists a disc $D$ centred at the point with the properties that $D$ does not meet $E$ and that $f^{-1}(D)$ contains mutually disjoint copies of $D$. Corresponding to each such disc $D$ let $B$ be the open disc concentric with $D$ that has radius one-third the radius of $D$. The collection of all such open discs $B$ then forms an open cover of the set $Q$.

Since $Q \cup E$ is a closed set in view of Lemma 2.3, $Q$ can be expressed as the countable union of a collection of compact subsets of $\mathbf{C}$. This implies that $Q$ has an open subcover of countably many $\operatorname{discs} B$. Let $V$ denote the union of all the discs in the subcover, and let $\mathcal{U}$ be any connected component of $V$; then $\mathcal{U}$ is a domain which is the union of some subcollection of the discs in the subcover. Let $\mathcal{B}$ denote this subcollection of discs $B$.

Let the $\operatorname{disc} B \in B$, and let $\tilde{B}$ be a copy of $B$ (cf. Definition 2.1). Then there is a branch of $f^{-1}$ that maps $B$ univalently onto $\tilde{B}$. Let $\left(f^{-1}, B\right)$ denote the function element determined by a branch of $f^{-1}$ on $B$. Since $B$ has exactly $q$ copies in B, there are exactly $q$ distinct such function elements; whenever we need to distinguish these, we will denote them as $\left(f_{1}^{-1}, B\right),\left(f_{2}^{-1}, B\right), \ldots,\left(f_{q}^{-1}, B\right)$.

In the remainder of this section we will use theory, definitions and notation from [11, pp. 316-319].

We will make much use of the following couple of self-evident lemmas which are given without proofs.

Lemma 3.1. Let $B, B^{\prime}$ be overlapping discs in $\mathcal{B}$. Then corresponding to each function element $\left(f^{-1}, B\right)$ there exists a unique function element $\left(f^{-1}, B^{\prime}\right)$ such that $\left(f^{-1}, B\right)$ and $\left(f^{-1}, B^{\prime}\right)$ are direct analytic continuations of each other.

For a fixed choice of a disc $B_{1}$ in $\mathcal{B}$ and a fixed function element $\left(f^{-1}, B_{1}\right)$, we deduce the following result.

Lemma 3.2. The function element $\left(f^{-1}, B_{1}\right)$ admits unrestricted analytic continuation in $\mathcal{U}$; that is, $\left(f^{-1}, B_{1}\right)$ can be continued analytically along every curve $\gamma$ in $\mathcal{U}$ that starts at the centre of $B_{1}$. Furthermore, the continuation of $\left(f^{-1}, B_{1}\right)$ along $\gamma$ ends in some function element $\left(f_{j}^{-1}, B\right)$, where $1 \leq j \leq q$ and $B$ is some disc in $\mathcal{B}$ that contains the endpoint of $\gamma$.

In general the set $\mathcal{U}$ may not be simply connected; nevertheless the following result holds, somewhat surprisingly. 
Lemma 3.3. The given function element $\left(f^{-1}, B_{1}\right)$ extends to a singlevalued (analytic) branch of $f^{-1}$ in $\mathcal{U}$.

Proof. Since every function element $\left(f^{-1}, B\right)$ in $\mathcal{U}$ admits unrestricted analytic continuation in $\mathcal{U}$, it follows, by standard arguments, that it suffices to show that if $g=\left(f^{-1}, B\right)$ is a function element in $\mathcal{U}, b$ is a point in $B$ and $\gamma$ is a simple closed arc in $\mathcal{U}$ which starts and ends at $b$, then the complete lift of $\gamma$ from the point $g(b)$ is a closed arc $\tilde{\gamma}$ and $f$ is injective in $\tilde{\gamma}$.

Since $f$ is finite-to-one and the continuation of $g$ along $\gamma$ is unrestricted and can be repeated, it follows that $\tilde{\gamma}$ is a closed arc. The injectivity of $f \mid \tilde{\gamma}$ follows by Lemma 2.1. This completes the proof.

We close this section with our second central lemma, concerning the union $V$ of open discs in a subcover of $Q$.

Lemma 3.4. The set $f^{-1}(V)$ contains $q$ mutually disjoint copies of $V$.

Proof. It is sufficient to prove the result for any given component $\mathcal{U}$ of $V$.

Let $\left(f_{1}^{-1}, B_{1}\right),\left(f_{2}^{-1}, B_{1}\right), \ldots,\left(f_{q}^{-1}, B_{1}\right)$ be the $q$ distinct function elements on $B_{1}$ that are induced by $f^{-1}$; by the previous lemma, each extends to an analytic function on $\mathcal{U}$ which is an inverse to $f$ on $\mathcal{U}$. If we denote by $f_{j}^{-1}$ the extension of $\left(f_{j}^{-1}, B_{1}\right)$ to $\mathcal{U}$, then $f_{j}^{-1}$ is univalent in $\mathcal{U}$ and $\tilde{\mathcal{U}}_{j}=f_{j}^{-1}(\mathcal{U})$ is a copy of $\mathcal{U}$. It remains to show that the copies $\tilde{\mathcal{U}}_{1}, \tilde{\mathcal{U}}_{2}, \ldots, \tilde{\mathcal{U}}_{q}$ are pairwise disjoint.

Suppose that in fact there is some point $z_{0}$ which belongs to $\tilde{\mathcal{U}}_{j_{1}} \cap \tilde{\mathcal{U}}_{j_{2}}$, where $j_{1} \neq j_{2}$. Let $w_{0}=f\left(z_{0}\right)$; and let $w_{1}$ be a point of $B_{1}$ that is different from $w_{0}$, and let $\alpha$ be an arc in $\mathcal{U}$ from $w_{1}$ to $w_{0}$. Since $f$ is locally univalent, it is easy to conclude that $f_{j_{1}}^{-1}$ and $f_{j_{2}}^{-1}$ must coincide on $\alpha$ and that $f_{j_{1}}^{-1}\left(w_{1}\right)=f_{j_{2}}^{-1}\left(w_{1}\right)$. This implies $\left(f_{j_{1}}^{-1}, B_{1}\right)=\left(f_{j_{2}}^{-1}, B_{1}\right)$, which is a contradiction. This completes the proof of the Lemma.

\section{The proof of Theorem 1}

In this section we prove Theorem 1 by showing how the image surface of a function $f$ satisfying the hypotheses $(p, q)$ can be extended to give a compact, analytic, $p$-sheeted covering of the Riemann sphere (as described in Theorem 1).

The image surface of $f$ is an analytic covering of the $w$-plane. We denote it by a pair $(Y, \pi)$ where $Y$ is the Riemann surface formed from pairs $(z, f(z))$, with the topological and conformal structures inherited from the unit disc $\mathbf{B}$ via $f$, and where $\pi$ is the natural projection map defined by $\pi(z, f(z))=f(z)$. The map from B onto $Y$ defined by $\tilde{f}(z)=(z, f(z))$ is conformal.

Let $G$ be an open subset of $\mathbf{C}$. It is easily seen that $G$ has a copy under $f$ if and only if it has a copy under $\pi$; and the latter is the image of the former under $\tilde{f}$. For convenience, we will use the same notation $\tilde{G}$ for both copies of $G$. Notice 
that facts about the copies under $f$ of the open sets $P$ and $V$, such as Lemmas 2.7 and 3.4, will carry over to their copies under $\pi$.

Now, by virtue of Lemma 3.4 there are exactly $q$ copies $\tilde{V}_{1}, \tilde{V}_{2}, \ldots, \tilde{V}_{q}$ of $V$ in $Y$. We will construct an additional $(p-q)$ copies as follows. For each $j$ with $q+1 \leq j \leq p$ let $\tilde{V}_{j}=\{(w, j): w \in V\}$. Let $X_{0}$ be the disjoint union of $Y$ and the sets $\tilde{V}_{q+1}, \tilde{V}_{q+2}, \ldots, \tilde{V}_{p}$. Then we extend $\pi$ to the "new copies" $\tilde{V}_{j}$ by the identification $\pi((w, j))=w$, and we topologize these copies by the topology that makes the restriction of $\pi$ to $\tilde{V}_{j}, q+1 \leq j \leq p$, a homeomorphism.

Next, let $G$ be any connected component of the open set $P \cap V$. Since $G$ is contained in $P$, it follows by Lemma 2.7 that $G$ has exactly $p$ copies in $Y$ of which only $q$ of them lie in $\tilde{V}_{1}, \tilde{V}_{2}, \ldots, \tilde{V}_{q}$. Denote the remaining copies by $\tilde{G}_{q+1}, \tilde{G}_{q+2}, \ldots, \tilde{G}_{p}$. Meanwhile every $\tilde{V}_{j}, q+1 \leq j \leq p$, contains a single copy $\hat{G}_{j}$ of $G$. Now for every $j, q+1 \leq j \leq p$, we identify points $M$ in $\tilde{G}_{j}$ with points $N$ in $\hat{G}_{j}$ if $\pi(M)=\pi(N)$, and we do this for all the components $G$ of $P \cap V$.

Now let $X$ be the quotient set of $X_{0}$ with the identification defined above. For simplicity, we may view $Y$ and each additional copy $\tilde{V}_{j}, q+1 \leq j \leq p$, as subsets of $X$. Similarly we may view $\pi$ as being defined on $X$. Then if $X_{0}$ is endowed with the free union topology, it follows that $X$ inherits the identification topology in which $Y$ and the additional copies $\tilde{V}_{j}, q+1 \leq j \leq p$, are open subsets of $X$ (see [4; pp. 120-136]).

It is immediate that $X$ is connected since $Y$ and each $\tilde{V}_{j}, q+1 \leq j \leq p$, are connected and they intersect in $X$. Observe from the construction of $X$ that no two points of $Y$ have been identified, and so also no two points of $\tilde{V}_{j}, 1 \leq j \leq p$. Hence for every $w \in \mathbf{C} \backslash E$ the set $\pi^{-1}(w)$ consists of exactly $p$ points in $X$. Using this, it can be easily verified that $X$ is Hausdorff, and that every point of $X$ lies in a neighbourhood which is homeomorphic under $\pi$ to an open disc in C. Therefore $S$ is a surface which with the latter local homeomorphisms as a conformal structure becomes a Riemann surface.

Now we make the pair $(X, \pi)$ a compact, analytic, $p$-sheeted covering of the Riemann sphere by attaching the appropriate number of points of $X$ over the exceptional points on $\infty$ (see [1; pp. 39-41]). This is done in the same manner as in the proof of the main theorem of Lyzzaik and Styer [6; p. 40], and the proof of Theorem 1 is complete.

\section{The proof of Theorem 2}

Let $P, Q$ and $E$ be as in Definition 1.1 and $X, Y$ and $\pi$ as in Section 4 . We shall perform a cutting-and-pasting exercise on $X$ that leads to a $p$-sheeted, compact, analytic covering $W$ of the Riemann sphere $\mathbf{P}$ such that the sum of the orders of the branch points over each point $e_{i}$ in $E$ is at most $\delta_{q}\left(e_{i}\right)$, and $Y$ is embedded in $W$.

If $E=\emptyset$, there is nothing to prove as Theorem 2 then reduces to Theorem 1 . 
(As we shall see below, in Section 6, actually this case cannot occur.) So let us assume that $E \neq \emptyset$.

Choose any element $e \in E$, and let $e^{\prime}$ be any other element of $E \cup\{\infty\}$. From our hypotheses there exists a simple arc, $\alpha$ say, that joins $e$ to $e^{\prime}$ in the set $Q \cup E \cup\{\infty\}$; for the moment we will suppose that $\alpha$ minus its endpoints lies in $Q$. In view of Lemma 3.4, it follows that there must exist $p-q$ simple $\operatorname{arcs} \tilde{\alpha}_{1}, \tilde{\alpha}_{2}, \ldots, \tilde{\alpha}_{p-q}$ in $X \backslash Y$ with mutually exclusive "interiors" such that each is a lift of $\alpha$ under $\pi$. It follows that $Y$ is not altered by cutting $X$ along the $\operatorname{arcs} \tilde{\alpha}_{j}$; but, of course, $X$ may separate. This possibility, however, cannot occur since every connected component of the slit surface $X \backslash\left(\cup \tilde{\alpha}_{j}\right)$ covers every point of $\mathbf{P} \backslash\left(\cup \alpha_{j}\right)$ at least once, hence the interior of the connected component of the slit surface that contains $Y$ covers under $\pi$ every point of $\mathbf{P}$ at most $(p-1)$ times contradicting the assumption on $f$.

Now suppose that $f$ covers the point $e$ exactly $v$ times. (Certainly we must have $v \leq q-1$, by Lemma 2.4.) Let $M$ be any point of $\pi^{-1}(e) \cap\{X \backslash Y\}$; if $b(M)$ denotes the branch order of $M$, then

$$
p-v=\sum_{M}(b(M)+1)
$$

where the sum is taken over all such points $M$.

Next, let $D$ be a topological disc satisfying $D \cap E=\{e\}$ and $D \cap \alpha=\beta$, where $\beta$ is a simple arc. It follows that $\pi^{-1}(D)$ is a disjoint union of topological discs, each of which covers $D$ under $\pi$ the same number of times. In particular, the disc $\tilde{D}_{M}$ that contains the point $M$ covers every point of $D$ exactly $b(M)+1$ times, taking account of multiplicity.

We now define $\tilde{\beta}_{j}=\tilde{\alpha}_{j} \cap \pi^{-1}(\beta)$ for each $j, 1 \leq j \leq p-q$; each $\tilde{\beta}_{j}$ is a lift of $\beta$, and lies in $\tilde{D}_{M}$ for some point $M$. Let $M$ be fixed; and suppose that $\tilde{D}_{M}$ contains more than one arc $\tilde{\beta}_{j}$-we choose to denote these $\operatorname{arcs}$ as $\tilde{\beta}_{j_{1}}, \tilde{\beta}_{j_{2}}, \ldots, \tilde{\beta}_{j_{s}}$ (where $s=s(j)$ and $1 \leq s \leq p-q)$ as we go about $M$ in $\tilde{D}_{M}$ in the positive direction. Next we cut $X$ along each arc $\tilde{\alpha}_{j_{k}}(1 \leq k \leq s)$, starting from $M$; and, for each such $k$, we agree to call the resulting right (left) edge the positive (negative) edge of $\tilde{\alpha}_{j_{k}}$. Then for each $k$ we identify crosswise the negative edge of $\tilde{\alpha}_{j_{k}}$ with the positive edge of $\tilde{\alpha}_{j_{(k+1)}}$, with the convenient convention that $\tilde{\alpha}_{j_{(s+1)}}=\tilde{\alpha}_{j_{1}}$. (Observe that this process "partitions" the disc $\tilde{D}_{M}$ into topological discs each of which, loosely speaking, contains a single $\operatorname{arc} \tilde{\beta}_{j}$.)

Continuing this process (over $e$ ) to all other such points $M$, as necessary, we obtain a $p$-sheeted, compact, analytic covering surface $W_{0}$ that contains $Y$; in terms of the notation associated with $X$ and which extends in the obvious way to $W_{0}$, the set $\pi^{-1}(D)$ consists of connected components each of which covers every point of $D$ the same number of times and contains at most one copy of the $p-q$ 
$\operatorname{arcs} \tilde{\beta}_{j}, 1 \leq j \leq p-q$. We then have that

$$
p-v=\sum_{M}(b(M)+1) \geq \sum_{M} b(M)+(p-q),
$$

which we rearrange as

$$
\sum_{M} b(M) \leq q-v \leq \delta_{q}(e)
$$

The surface $W_{0}$ contains $Y$ and has the desired bound on the sum of branch orders over one (arbitrary) point of $E$. We have now to show how we can apply such a construction repeatedly over (all) the points of $E$, so that the resulting surface contains $Y$ and has the desired bound on the sum of branch orders over every point of $E$.

To do this, we use the fact that any two points $e, e^{\prime}$ in the set $E \cup\{\infty\}$ are (by hypothesis) arcwise connected in $Q \cup E \cup\{\infty\}$. First, we partition $E$ inductively into finitely many subsets $E_{k}$, as follows. Let $E_{1}$ be the set of all points $e \in E$ such that $e$ and $\infty$ are connected by an arc $\alpha$ in $Q \cup E \cup\{\infty\}$ such that the "open" arc $\alpha$ does not meet $E$. Suppose that, for some $k \geq 1, E_{k}$ has been defined and that $\bigcup_{s=1}^{k} E_{j}$ does not exhaust $E$. Then we define $E_{k+1}$ to be the set of all points $e$ of $E \backslash\left\{\bigcup_{j=1}^{k} E_{j}\right\}$ such that $e$ and some point $e^{\prime}$ of $E_{k}$ can be connected by an arc $\alpha$ in $Q \cup E \cup\{\infty\}$ such that $\alpha$ minus its endpoints does not meet $E$. Then clearly $E_{k+1}$ is non-empty; and there must be some positive integer $N$ such that $E_{1}, E_{2}, \ldots, E_{N}$ are non-empty and form a partition of $E$. Furthermore, corresponding to each point $e_{i}$ of $E_{k}$, where $1 \leq i \leq r$ and $1 \leq k \leq N$, there is some simple arc $\alpha_{i}$ that joins $e_{i}$ to some point of $E_{k-1}$ within $Q$, except for its endpoints. (Here we may, without loss of generality, take $E_{0}=\{\infty\}$.)

We may therefore assume that $X$ has been chosen so that the sum of the branch orders of $X$ over each point $e_{i}, 1 \leq i \leq r$, does exceed $\delta_{q}\left(e_{i}\right)$, and so that the ordering of the points $\left\{e_{i}\right\}$ of $E$ satisfies the following relation: If $i<j$, then there are integers $\mu, \nu$, with $1 \leq \mu \leq \nu \leq s$, such that $e_{i} \in E_{\nu}$ and $e_{j} \in E_{\mu}$. We construct $W$ by showing that there is a sequence $W_{1}, W_{2}, \ldots, W_{r}$ of $p$-sheeted, compact, analytic coverings of $\mathbf{P}$ such that each covering $W_{i}$ contains $Y$ and has the sum of orders of its branch points over each $e_{j}, 1 \leq j \leq i$, at most $\delta_{q}\left(e_{j}\right)$.

This can be achieved by induction on $i$, as follows. We construct $W_{1}$ from $X$ in the same way as we constructed $W_{0}$, by taking $e_{1}$ and $\alpha_{1}$ for $e$ and $\alpha$, respectively. So now suppose that, for some $i$ with $1 \leq i<r$, we have constructed the surface $W_{i}$ with the desired properties. Then we can construct $W_{i+1}$ from $W_{i}$ just as we constructed $W_{0}$ from $X$ using $e_{i+1}$ and $\alpha_{i+1}$ for $e$ and $\alpha$, respectively. Since $\alpha_{i+1} \cap\left\{e_{1}, e_{2}, \ldots, e_{i}\right\}=\emptyset$, it follows that $W_{i+1}$ and $W_{i}$ coincide locally over each point $e_{j}$ with $1 \leq j \leq i$. Thus $W_{i+1}$ has the desired properties.

This completes the inductive construction, and $W_{r}=W$ as desired. 
We close this section by conjecturing that Theorem 2 holds without the extra assumption of arcwise connectedness on the set $E \cup\{\infty\}$ in $Q \cup E \cup\{\infty\}$.

\section{Consequences - the analytic case}

In this section we study the consequences of Theorems 1 and 2 for functions satisfying the hypotheses $(p, q)$; in particular, the connection between the cardinality, $r$, of the exceptional set $E$ and the deficiency, $\delta_{q}(f)$, of $f$.

Recall that $E=\left\{e_{1}, e_{2}, \ldots, e_{r}\right\}, \delta_{q}(f)=\sum_{i=1}^{r} \delta_{q}\left(e_{i}\right)$, and the coverings $X$ and $W$ arising in Theorems 1 and 2. Whether dealing with $X$ or $W$, we define $q\left(e_{i}\right)$ and $q(\infty)$ to be the sum of the orders of those branch points lying over $e_{i}$ and $\infty$, respectively. Then $q(\infty) \leq p-1$, with equality if and only if the associated surface has a single branch point over $\infty$ of order $(p-1)$.

We have the following result

Theorem 3. Let the function $f$ satisfy the hypotheses $(p, q)$, and let $E=\left\{e_{1}, \ldots, e_{r}\right\}$. Then the deficiency of $f$ satisfies the inequality

$$
r(p-q-1)+\delta_{q}(f) \geq p-1 .
$$

In particular, $r>0$.

If equality holds in (*), then the covering surface $X$ of $\mathbf{P}$ that embeds the Riemann surface $Y$ of the image of $f$ is the image Riemann surface of a polynomial of degree $p$. That is, $f=H \circ \varphi$, where $H$ is a polynomial in $\mathbf{C}$ of degree $p$ and $\varphi$ is analytic and univalent in $\mathbf{B}$.

Proof. For every point $e_{i}$ in $E$, the surfaces $X$ and $W$ have at least card $f^{-1}\left(e_{i}\right)$ smooth sheets over $e_{i}$. In fact, there are card $f^{-1}\left(e_{i}\right)$ of these sheets in $Y$. Accordingly, if in $X$ there are exactly $1+\operatorname{card} f^{-1}\left(e_{i}\right)$ points over $e_{i}$, then $q\left(e_{i}\right)=p-q+\delta_{q}\left(e_{i}\right)-1$; otherwise $q\left(e_{i}\right) \leq p-q+\delta_{q}\left(e_{i}\right)-1$.

We proceed by applying the Riemann-Hurwitz relation (see, for example, [5; p. 16] to $X$. The genus, $g$, of $X$ is given by the formula

$$
g=\frac{1}{2}\left(q(\infty)+\sum_{i=1}^{r} q\left(e_{i}\right)\right)-p+1,
$$

where $g$ is some non-negative integer, $q(\infty) \leq p-1$ and

$$
\sum_{i=1}^{r} q\left(e_{i}\right) \leq r(p-q-1)+\delta_{q}(f) .
$$

From these facts the inequality $(*)$ follows at once.

Suppose that $r$ equalled zero. Then from the definition of deficiency we must have $\delta_{q}(f)=0$, which would mean that $(*)$ became the inequality $0 \geq p-1$. This contradiction shows that $r>0$. 
Suppose next that equality holds in $(*)$. It follows from the above relations that $g$ must vanish, so that by the uniformization theorem $[13 ;$ p. 225] $X$ is conformally equivalent to the Riemann sphere $\mathbf{P}$, and $X$ must be the Riemann surface of the image of a polynomial $H$ of degree $p$. Since the Riemann surface, $Y$, of the image of $f$ is embedded in $X$, it follows that $f=H \circ \psi$ where $\psi$ is analytic univalent, as desired.

Remark 6.1. The equality in (*) is attained whenever $q=p-1$ (see Example 6.1 below); we conjecture that, if $q<p-1$, then equality cannot be attained. The truth of this conjecture is supported by Theorem 4 below.

Corollary 6.1. If, under the hypotheses of Theorem 3, each deficiency $\delta_{q}\left(e_{i}\right)$ is equal to $s$, say, then

$$
r \geq \frac{p-1}{p+s-q-1}
$$

Corollary 6.2. If, under the hypotheses of Theorem $3, q=p-1$, then we must have

$$
\delta_{q}(f) \geq p-1 .
$$

This is the result of Lyzzaik and Styer [6; p. 42] that proved an earlier conjecture of Brannan and Kirwan [2; top of p. 100].

Next we consider consequences of Theorem 2. It follows from Theorem 2 that $q\left(e_{i}\right) \leq \delta_{q}\left(e_{i}\right)$ for each point $e_{i}$ of $E$. Using this fact and the method of proof of Theorem 3 , we can prove the following

Theorem 4. Let the function $f$ satisfy the hypotheses $(p, q)$; assume, in addition, that any two points of $E \cup\{\infty\}$ are arc-wise connected in $Q \cup E \cup\{\infty\}$. Then $\delta_{q}(f) \geq p-1$. If $\delta_{q}(f)=p-1$, then $f=H \circ \varphi$, where $H$ is a polynomial in $\mathbf{C}$ of degree $p$ and $\varphi$ is analytic and univalent in $\mathbf{B}$.

The following special case of Theorem 4 is of sufficient interest to be stated independently.

Corollary 6.3. Under the assumptions of Theorem 4, if every exceptional point is covered by $f$ zero times, then we must have that $r \geq(p-1) / q$.

Corresponding to the case $q=0$ of Theorem 3 we have the following.

Remark 6.2. If $f$ is analytic and locally univalent in $\mathbf{B}$ and $v(f)=\{p, 0\}$ then $p=1$.

Since each connected component of $P$ is simply connected (by Lemma 2.3) and $P=f(\mathbf{B})$ is connected, $P$ is a simply connected domain. But by Lemma 2.7, $f^{-1}(P)$ consists of $p$ mutually disjoint copies $\tilde{P}_{1}, \tilde{P}_{2}, \ldots, \tilde{P}_{p}$ of $P$. Thus

$$
\mathbf{B}=\bigcup_{j=1}^{p} \tilde{P}_{j}
$$


from which it follows that $p=1$.

We end this section with an example which shows that equality in Theorem 3 is attained if $q=p-1$, and that equality in Theorem 4 is attained for every $q$. It generalizes Example 2 in [2], where $q$ is taken to be $(p-1)$.

Example 6.1. Let $p>q \geq 1$ be positive integers. There exists an analytic univalent function $\varphi$ in $\mathbf{B}$ and a polynomial $g$ of degree $p$ such that $f=g \circ \varphi$ satisfies the hypotheses $(p, q)$ and $\delta_{q}(f)=p-1$. We show this by constructing the image surface of $f$.

Let $r$ be a positive integer; and, for each $i$ with $1 \leq i \leq r$, let $e_{i}$ be a (distinct) point in $\mathbf{C}, v\left(e_{i}\right)$ a non-negative integer, and $d_{i}$ a (Euclidean) ray with initial point $e_{i}$. Assume further that $v\left(e_{i}\right) \leq q-1, \sum_{i=1}^{r} \delta_{q}\left(e_{i}\right)=p-1$ where $\delta_{q}\left(e_{i}\right)=q-v\left(e_{i}\right)$, and that the rays $d_{i}$ are mutually disjoint.

Let $\mathbf{C}_{1}, \mathbf{C}_{2}, \ldots, \mathbf{C}_{p}$ be $p$ copies of $\mathbf{C}$. For convenience we make the definition

$$
\delta_{k}= \begin{cases}1, & \text { if } k=0 \\ 1+\sum_{i=1}^{k} \delta_{q}\left(e_{i}\right) & \text { if } 1 \leq k \leq r-1\end{cases}
$$

Now for each $k$ with $0 \leq k \leq r-1$, cut $\mathbf{C}_{\delta_{k}}, \mathbf{C}_{\delta_{k}+1}, \ldots, \mathbf{C}_{\delta_{k+1}}$ along the ray $d_{k+1}$; this procedure ensures that every copy $C_{\delta_{k}}$, with $1 \leq k \leq r-1$, is cut twice, once along $d_{k}$ and once along $d_{k+1}$.

Next we sew together the slit planes in the following way. For each $k$ with $0 \leq k \leq r-1$, sew the copies $\mathbf{C}_{\delta_{k}}, \mathbf{C}_{\delta_{k}+1}, \ldots, \mathbf{C}_{\delta_{k+1}}$ crosswise along the edges of the cuts over $d_{k+1}$ in such a way that a branch point of order $\delta_{q}\left(e_{k+1}\right)$ is obtained over the point $e_{k+1}$. This gives a $p$-sheeted analytic covering of $\mathbf{C}$ with branch points of order $\delta_{q}\left(e_{i}\right)$ over $e_{i}, 1 \leq i \leq r$; let us call this surface $X$.

Then we compactify this surface in the usual way by filling up the missing points over $\infty$. This may lead to branch points over $\infty$, sum of whose orders is at most $(p-1)$. Since $\sum_{i=1}^{r} \delta_{q}\left(e_{i}\right)=p-1$, the sum of all the orders of the branch points of $X$ (which now covers all of $\mathbf{P}$ ) is at most $(2 p-2)$. But by the Riemann-Hurwitz formula [5; p. 16] $X$ has only one branch point over $\infty$ of order $p-1$ and is simply connected. Hence by the uniformization theorem [13; pp. 58-59] $X$ is the image surface of a polynomial of degree $p$.

Finally, for each $i$ with $1 \leq i<r$ we cut the surface $X$ over the ray $d_{i}$ exactly $(p-q)$ times; one and only one cut is taken through the branch point over $e_{i}$, and the rest elsewhere. If we now denote the resulting surface by $Y$, then $Y$ is simply connected. Since $Y$ is clearly hyperbolic, it follows from the uniformization theorem that there exists an analytic function $f: \mathbf{B} \rightarrow \mathbf{C}$ whose image surface is $Y$. From the construction of $Y$, it easily follows that $f$ has the desired properties. 


\section{Consequences - the continuous locally-univalent case}

In this section we discuss results following from Theorems 3 and 4 , where the assumption of analyticity can be replaced by continuity together with local injectivity.

First we obtain a generalization of Theorem 3 with $\mathbf{B}$ and $\mathbf{C}$ being replaced by simply-connected domains $\Omega_{1}$ and $\Omega_{2}$, respectively, and with $f$ being assumed to be a continuous, locally one-one function from $\Omega_{1}$ to $\Omega_{2}$ such that every point of $\Omega_{2}$ is covered by $f$ either $p$ times or $q$ times with finitely many exceptional points $e_{1}, e_{2}, \ldots, e_{r}$ of $\Omega_{2}$.

Since a simply-connected domain is homeomorphic to both $\mathbf{B}$ and $\mathbf{C}$, we may assume without loss of generality that $\Omega_{1}=\mathbf{B}$ and $\Omega_{2}=\mathbf{C}$. Then it follows from Riemann surface considerations that $f=\psi \circ \varphi$ where $\varphi$ is a homeomorphism of $\mathbf{B}$ onto $\mathbf{B}$ and $\psi$ is a function analytic in $\mathbf{B}$ that covers every point of $\mathbf{C}$ either $p$ times or $q$ times, with the exception of the points $e_{1}, e_{2}, \ldots, e_{r}$. This implies that each exceptional point $e_{i}$ is covered by $\psi$ at most $(q-1)$ times, by Lemma 2.4; and, consequently, by the decomposition $f=\psi \circ \varphi$ each point $e_{i}$ is also covered by $f$ at most $(q-1)$ times. Accordingly, we may define the deficiency, $\delta_{q}(w)$, of $f$ at points $w$ of $\Omega_{2}$ in the usual way, and similarly the deficiency of $f$ (in $\Omega_{1}$ ) to be $\delta_{q}(f)=\sum \delta_{q}(w)$ where the sum is taken over all $w \in \Omega_{2}$, and the $q$-deficiency of $f$ in $\Omega_{1}$ is $\delta_{q}(f)=\sum_{i=1}^{r} \delta_{q}\left(e_{i}\right)$.

If we apply Theorem 3 to the function $\psi$, and then pull the results back to $f$ via $\varphi$, we obtain the following analog of Theorem 3 .

Corollary 7.1. Let $\Omega_{1}, \Omega_{2}$ be simply connected domains in $\mathbf{C}$, and let $f$ be a continuous locally one-one function from $\Omega_{1}$ to $\Omega_{2}$ such that each point of $\Omega_{2}$ is covered by $f$ either $p$ times or $q$ times $(p>q>0)$, with exceptions at the points $e_{1}, e_{2}, \ldots, e_{r}$ in $\Omega_{2}$. If $\delta_{q}(f)$ is the $q$-deficiency of $f$ in $\Omega_{1}$, then the following inequality holds:

$$
r(p-q-1)+\delta_{q}(f) \geq p-1
$$

In particular, $r>0$.

A consequence of this result is the following, which settles in the affirmative a recent conjecture of Ortel and Smith [8].

Corollary 7.2. There is no continuous locally one-one function between any two simply-connected domains such that every point of the image domain is covered by the function either $p$ times or $q$ times.

Notice that the requirement of local injectivity in Corollary 7.2. is essential, as the following example shows. 
Example 7.1. Let $P$ be a polynomial of precise degree $p$, all of whose zeros lie in $\mathbf{B}$, such that $P^{\prime}$ has simple zeros (necessarily all in B). Let $X$ be the Riemann surface of the function $P$ over $\mathbf{C}$. Now slit $X$ from the point 1 radially outward to $\infty$ along $(p-q)$ sheets of $X$, to obtain a new Riemann surface $Y$. Then clearly there exists some function $f$, analytic in $\mathbf{B}$, that maps $\mathbf{B}$ one-one onto $Y$, and for which $v(f)=\{p, q\}$.

An immediate consequence of Corollary 7.2. that is of interest in its own right is the following curious result.

Corollary 7.3. Let the function $f: \mathbf{B} \rightarrow \mathbf{C}$ be continuous, locally one-one, and onto. Then if $\operatorname{card}(v(f)) \neq 1$, we must have that $\operatorname{card}(v(f)) \geq 3$.

We continue our discussion with the function $f: \Omega_{1} \rightarrow \Omega_{2}$ introduced in Corollary 7.1. Our next result is the analog of Theorem 4 for such a function. We extend the definitions of $P, Q$ and $E$ (see Definition 1.1) to this function in the natural manner, and we define $e_{\infty}=\mathbf{P} \backslash \Omega_{2}$. Then we can use the ideas in Section 2 that lead to Lemma 2.6 to prove that the set $Q \cup E \cup e_{\infty}$ must be connected.

In addition, we will assume that the function $f$ satisfies the following stronger condition.

Condition A. There exists open arcs $\alpha:] 0,1[\rightarrow Q$ such that

(i) the set union of these arcs and $E \cup\left\{e_{\infty}\right\}$ is connected, and

(ii) the cluster sets, $C(\alpha, 0)$ and $C(\alpha, 1)$, of every arc $\alpha$ at 0 and 1, respectively, are subsets of different sets $e_{j}, j=1,2, \ldots, r$ and $\infty$.

Clearly Condition A, the hypotheses on $f$, and $\delta_{q}(f)$ are all invariant under homeomorphism on $\Omega_{1}$ and $\Omega_{2}$. Thus without loss of generality we may assume that $\Omega_{1}=\mathbf{B}$ and $\Omega_{2}=\mathbf{C}$.

Under the latter assumption, Condition A says that any two points in $E \cup\{\infty\}$ are arc-wise connected in $Q \cup E \cup\{\infty\}$. It follows from the above decomposition $f=\psi \circ \varphi$ where $\varphi: \mathbf{B} \rightarrow \mathbf{B}$ is a homeomorphism that $\psi$ inherits the above requirements on $f$, and also that $\delta_{q}(\psi)=\delta_{q}(f)$. Moreover, $\psi$ is analytic and locally univalent in B. Therefore by Theorem $4, \delta_{q}(\psi) \geq p-1$; from which we deduce the following result:

Corollary 7.4. Let $\Omega_{1}$ and $\Omega_{2}$ be simply connected domains in $\mathbf{C}$, and let $f$ be a continuous locally one-one function from $\Omega_{1}$ to $\Omega_{2}$ such that each point of $\Omega_{2}$ is covered by $f$ either $p$ times or $q$ times $(p>q>0)$, with exceptions at the points $e_{1}, e_{2}, \ldots, e_{r}$ in $\Omega_{2}$. Suppose further that $f$ satisfies Condition (A). Then $\delta_{q}(f) \geq p-1$.

Our final result in this section establishes a connection between the covering properties of a function and the connectivity of its image domain.

Let $G$ be a domain in the plane with connectivity $n$ (this is the number of bounded connected components of $\mathbf{C} \backslash G$ ), and let $f: \mathbf{B} \rightarrow G$ be a continuous 
locally one-one function that covers every point of $G$ either $p$ times or $q$ times. We find below the 'best possible' lower bound for $n$ in the case that $q=p-1$, and also in some other cases under a supplementary condition (Condition B below) imposed on $f$.

Corollary 7.5. Let $f$ have the above described properties. Then $n \geq 1$, and this is best possible in the case when $q=p-1$.

Proof. It follows from Corollary 7.2 that $n$ cannot vanish, and so $n \geq 1$.

For any positive integer $p$, the function $f(z)=((1+z) /(1-z))^{2 p}$ covers in B every point of $\Omega=\mathbf{C} \backslash\{0\}$ either $p$ times or $(p-1)$ times and it never covers 0 . This function has $q=p-1$, and $f(\mathbf{B})$ is of connectivity 1 ; thus the inequality of the theorem is best possible.

Suppose next that $q<p-1$. Let $Q$ denote the set of points of $G$ that are covered by $f$ exactly $q$ times; and let $F_{1}, F_{2}, \ldots, F_{n}, F_{n+1}$ denote the connected components of $\mathbf{P} \backslash G$, where $F_{n+1}$ is the unbounded connected component. Then an argument similar to that of Lemma 2.6 shows that the set $Q \cup F_{1} \cup F_{2} \cup \ldots \cup F_{n+1}$ is connected and each connected component of the set $Q \cup F_{1} \cup F_{2} \cup \ldots \cup F_{n}$ is not separated from $F_{n+1}$.

We now make the additional assumption that $Q$ satisfies the following:

Condition B. There exist open $\operatorname{arcs} \alpha:] 0,1[\rightarrow Q$ such that

(i) the set union of these arcs and $\sum_{j=1}^{n+1} F_{j}$ is connected, and

(ii) the cluster sets $C(\alpha, 0), C(\alpha, 1)$ of each arc $\alpha$ at its endpoints are subsets of different sets $F_{j}, 1 \leq j \leq n+1$.

Next, let $a_{1}, a_{2}, \ldots, a_{n}$ be any $n$ distinct points of $\mathbf{C}$. Then there exists a homeomorphism $h$ between $G$ and $\Omega=\mathbf{C} \backslash\left\{a_{1}, a_{2}, \ldots, a_{n}\right\}$ (see, for example, [4; p. 57]); consequently the function $F=h \circ f: \mathbf{B} \rightarrow \Omega$ is a continuous locally one-one function that covers every point of $\Omega$ exactly $p$ times or $q$ times. Notice that $F$ does not cover any point of $\left\{a_{1}, a_{2}, \ldots, a_{n}\right\}$, which we may equivalently view as the fact that $\delta_{q}\left(a_{i}\right)=p-1,1 \leq i \leq n$.

It is easy to see that Condition B implies that the points $a_{1}, a_{2}, \ldots, a_{n}$, $\infty$ are arc-wise connected in the set $h(Q) \cup\left\{a_{1}, a_{2}, \ldots, a_{n}, \infty\right\}$. Now let $\delta_{q}(F)$ be the $q$-deficiency of $F$ in $\mathbf{C}$, so that from our construction above we have that $\delta_{q}(F)=n q$. It follows from Corollary 7.4 that $n q \geq p-1$, so that

$$
n \geq(p-1) / q \text {. }
$$

We can summarize these results as follows.

Corollary 7.6. Let $G$ be a domain in $\mathbf{C}$ of connectivity $n, n>0$, and let $f: \mathbf{B} \rightarrow G$ be a continuous locally one-one function that covers every point of $G$ either $p$ times or $q$ times. If the set $Q$ satisfies Condition B then $n \geq(p-1) / q$, and this is best possible if $q$ divides $(p-1)$. 
The fact that the estimate is best possible will follow immediately from functions that we construct in Theorem 6 in Section 8.

\section{Possible sets for $v(f)$}

A sequence $p_{1}<p_{2}<\cdots<p_{n}, n \geq 3$, of non-negative integers is said to be a valence sequence if there exists a function $f$ analytic and locally univalent in $\mathbf{B}$ such that $f$ covers every point in $\mathbf{C} p_{j}$ times for some $j$, and such that for each $j$ there exists some point in $\mathbf{C}$ that is covered exactly $p_{j}$ times; in other words, $v(f)=\left\{p_{1}, p_{2}, \ldots, p_{n}\right\}$. The sequence $\left\{p_{j}\right\}_{1}^{n}$ is called the valence sequence of $f$.

In view of the results by many authors described in Section 1 and also presented here in Section 5, it would clearly be of great interest to identify those sequences that are valence sequences. While this remains an open question, we supply below two criteria that ensure that a given sequence is a valence sequence. In each case we do this by exhibiting an appropriate Riemann surface for such a function $f$.

Theorem 5. Every sequence $p_{1}<p_{2}<\cdots<p_{n}$ of non-negative integers satisfying $p_{n}=p_{n-1}+1$ is a valence sequence of a function $f: \mathbf{B} \rightarrow \mathbf{C}$ which is a polynomial of degree $p_{n}$ of a univalent function.

Proof. First we will prove the result in the special case that $n=3$. (Note that we cannot have $n=2$, by Corollary 7.3; and the case $n=1$ is clearly possible for an elementary one-one function.)

If $p_{1}=0$ the result follows at once using the function construction in the proof of Corollary 7.5.

Now suppose that $p_{1}>0$. By the division algorithm, there must exist a positive integer $N$ such that

$$
p_{1}<N\left(p_{2}-p_{1}\right) \leq p_{2} .
$$

Then either (i) $N\left(p_{2}-p_{1}\right)=p_{2}$ or else (ii) $N\left(p_{2}-p_{1}\right)<p_{2}$. For convenience we will use the notation

$$
\alpha(N)= \begin{cases}N, & \text { if case (i) holds } \\ N+1, & \text { if case (ii) holds }\end{cases}
$$

Let $d_{1}, d_{2}, \ldots, d_{\alpha(N)}$ be mutually disjoint radial rays that do not pass through the origin and whose initial points are $w_{1}, w_{2}, \ldots, w_{\alpha(N)}$, respectively. Then we cover $\mathbf{C}$ by the copies $\mathbf{C}_{1}, \mathbf{C}_{2}, \ldots, \mathbf{C}_{p_{3}}$. Next, for each $j$ with $0 \leq j \leq$ $N-1$, we cut each of the copies $\mathbf{C}_{j\left(p_{2}-p_{1}\right)+1}, \mathbf{C}_{j\left(p_{2}-p_{1}\right)+2}, \ldots, \mathbf{C}_{(j+1)\left(p_{2}-p_{1}\right)+1}$ along the ray over $d_{j+1}$.

If case (i) holds, we have then exhausted cutting of all the $p_{3}$ sheets; if case (ii) holds, we make more cuts on the remaining copies $\mathbf{C}_{N\left(p_{2}-p_{1}\right)+1}, \mathbf{C}_{N\left(p_{2}-p_{1}\right)+2}, \ldots$, 
$\mathbf{C}_{p_{3}}$ along the ray over $d_{N+1}$. Then, for each $j$ with $0 \leq j \leq N-1$ we identify the copies $\mathbf{C}_{j\left(p_{2}-p_{1}\right)+1}, \mathbf{C}_{j\left(p_{2}-p_{1}\right)+2}, \ldots, \mathbf{C}_{(j+1)\left(p_{2}-p_{1}\right)+1}$ crosswise along the edges of the cuts over $d_{j+1}$; this process yields a branch point of order $\left(p_{2}-p_{1}\right)$ over $w_{j+1}$. If case (i) holds, we have thus joined up all the $p_{3}$ copies of $\mathbf{C}$; if case (ii) holds, we continue sewing together the remaining copies crosswise along the edges of the cuts over $d_{N+1}$ in such a way as to obtain a branch point of order $p_{3}-N\left(p_{2}-p_{1}\right)-1$ over $w_{N+1}$.

It is then easy to see that in either case this procedure yields a simply connected, $p$-sheeted covering $X$ of $\mathbf{C}$; and this covering is the image surface of a polynomial of degree $p_{3}$. This surface has branch points only over $w_{1}, w_{2}, \ldots$, $w_{\alpha(N)}$; and these points have the same order $\left(p_{2}-p_{1}\right)$ except in case (ii), where the branch point over $w_{N+1}$ has order $p_{3}-N\left(p_{2}-p_{1}\right)$.

Next, for each $j$ with $1 \leq j \leq \alpha(N)$, we cut $X$ radially along a ray over $d_{j}$ and through the branch point of $X$ over $w_{j}$. In case (ii) where $N\left(p_{2}-p_{1}\right)>p_{1}$, let $l_{1}, l_{2}, \ldots, l_{N\left(p_{2}-p_{1}\right)-p_{1}}$ be a pencil of (closed) rays starting from $w_{N+1}$ such that none of the $l_{i}$ or $d_{j}$ meet, except possibly at their end points; we then also cut each copy $\mathbf{C}_{j}$, with $1 \leq j \leq N\left(p_{2}-p_{1}\right)-p_{1}$, along the ray over $l_{j}$. In either case, let $Y$ denote the resulting surface.

It is fairly easy to see that $Y$ is a simply connected surface that covers every point $w_{j}$, with $1 \leq j \leq \alpha(N)$, exactly $p_{1}$ times. Furthermore, if case (i) holds, then $Y$ covers every point of $\mathbf{C} \backslash \bigcup_{j=1}^{N} d_{j}$ exactly $p_{3}$ times, and every point of $\bigcup_{j=1}^{N} d_{j}$ exactly $p_{2}$ times; if case (ii) holds, then $Y$ covers every point of $\mathbf{C} \backslash\left(\left(\bigcup_{j=1}^{N+1} d_{j}\right) \bigcup\left(\bigcup_{j=1}^{N\left(p_{2}-p_{1}\right)-p_{1}} l_{j}\right)\right)$ exactly $p$ times, and every point of $\left(\bigcup_{j=1}^{N+1} \operatorname{Int} d_{j}\right) \cup\left(\bigcup_{j=1}^{N\left(p_{2}-p_{1}\right)-p_{1}} \operatorname{Int} l_{j}\right)$ exactly $p_{2}$ times. Then if $f: \mathbf{B} \rightarrow \mathbf{C}$ is a function whose image is $Y$, it follows that $v(f) \quad\left\{p_{1}, p_{2}, p_{3}\right\}$.

Finally we consider the case that $n>3$. To begin with we construct, as described above, a surface $Y$ which is the image surface of a function whose valence is $\left\{p_{n-2}, p_{n-1}, p_{n}\right\}$. This surface covers mutually disjoint radial rays $\gamma_{j}$, $1 \leq j \leq n-3$, such that each is covered by $Y$ exactly $p_{n}$ times; for each such $j$, if we cut $Y$ along $p_{n}-p_{j}$ rays over $\gamma_{j}$ the resulting surface $Z$ is the image surface of the desired function $f$.

This completes the proof.

Notice that the function $f$ of Theorem 5 covers every point of $\mathbf{C}$ exactly $p_{n}$ times, except for points belonging to finitely many rays, disjoint except possibly for their endpoints, which can be chosen almost 'at random'.

Theorem 6. A sequence $p_{1}, p_{2}, \ldots, p_{n}$ of non-negative integers is a valence sequence if for some fixed integer $k$ (with $2 \leq k \leq n-1$ ) there is a sequence of 
non-negative integers $n_{1}, n_{2}, \ldots, n_{k-1}$ such that

$$
\sum_{j=1}^{k-1} n_{j}\left(p_{k}-p_{j}\right)=p_{n}-1 .
$$

In particular, when (**) holds the sequence is the valence sequence of a function $f$ which is a polynomial (of degree $p_{n}$ ) of a univalent function.

Proof. We establish the result by simply constructing a suitable Riemann surface, and then appealing to the uniformization theorem in the usual way.

Some of the integers $\left\{n_{j}\right\}_{j=1}^{k-1}$ are positive and some are zero; let us assume, for the moment, that all are positive-we will see at the end of our construction what changes we have otherwise to make.

Let $N=\sum_{j=1}^{k-1} n_{j}$.

Now choose distinct points $w_{1}, w_{2}, \ldots, w_{N}$ in $\mathbf{C}$, and mutually disjoint closed rays $d_{1}, d_{2}, \ldots, d_{N}$ taken radially from these points, respectively, with no ray passing through the origin. We now construct a suitable covering of $\mathbf{C}$ by copies $\mathbf{C}_{1}, \mathbf{C}_{2}, \ldots, \mathbf{C}_{p_{n}}$ of itself.

Let $t, \tau$ be integers satisfying the inequalities $0 \leq t \leq r-1$ and $0 \leq \tau \leq n_{t+1}$. We then define two numbers $\alpha(t, \tau), \beta(t, \tau)$ by the formulas

$$
\begin{gathered}
\alpha(t, \tau)= \begin{cases}1+\tau\left(p_{k}-p_{1}\right), & \text { if } t=0, \\
1+\tau\left(p_{k}-p_{t+1}\right)+\sum_{i=1}^{t} n_{i}\left(p_{k}-p_{i}\right), & \text { if } t>0,\end{cases} \\
\beta(t, \tau)= \begin{cases}1+\tau, & \text { if } t=0, \\
1+\tau+\sum_{i=1}^{t} n_{i}, & \text { if } t>0 .\end{cases}
\end{gathered}
$$

For fixed $t$ and all $\tau$ with $0 \leq \tau^{\cdot}<n_{t+1}$, we cut each of the copies $\mathbf{C}_{\alpha(t, \tau)}$, $\ldots, \mathbf{C}_{\alpha(t, \tau+1)}$ along the ray over $d_{\beta(t, \tau)}$, and then we sew together the copies crosswise along sides of the edges in such a way that we obtain a branch point of order $p_{k}-p_{t+1}$ over each point $w_{\beta(t, \tau)}$.

Then we repeat this construction for each $t$ with $0 \leq t \leq r-1$. Note that, except for the copy $\mathbf{C}_{1}=C_{\alpha(0,0)}$, every copy $\mathbf{C}_{\alpha(t, \tau)}$ (with $0 \leq t \leq r-1$ and $\left.0<\tau<n_{t+1}\right)$ has been cut twice along the rays $d_{\beta(t, \tau-1)}$ and $d_{\beta(t, \tau)}$. This serves via the above sewing to adjoin (for all $t$ and $\tau$ with $0 \leq \tau \leq n_{t+1}-2$ ) the 'stacks' $\mathbf{C}_{\alpha(t, \tau)}, \ldots, \mathbf{C}_{\alpha(t, \tau+1)}$ and $\mathbf{C}_{\alpha(t, \tau+1)}, \ldots, \mathbf{C}_{\alpha(t, \tau+2)}$, and (for all $t$ with $0 \leq t \leq r-2)$ the stacks $\mathbf{C}_{\alpha\left(t, n_{t+1}-1\right)}, \ldots, \mathbf{C}_{\alpha(t+1,0)}$ and $\mathbf{C}_{\alpha(t+1,0)}, \ldots$, $\mathbf{C}_{\alpha(t+1,1)}$.

It is fairly routine to check that this construction yields a simply connected $p_{n}$-sheeted covering of $\mathbf{C}$. We then make this into a Riemann surface $X$ in the usual way; and, by the uniformization theorem, $X$ is the image surface of a polynomial of degree $p_{n}$. This surface has branch points only over $w_{1}, w_{2}, \ldots, w_{N}$; and the order of the branch point over each point $w_{\beta(t, \tau)}$ (where $0 \leq t \leq r-1$ 
and $\left.0 \leq \tau<n_{t+1}\right)$ is exactly $p_{k}-p_{t+1}$. Furthermore, $w_{\beta\left(t, \tau_{1}\right)}=w_{\beta\left(t, \tau_{2}\right)}$ for fixed $t$ and $0 \leq \tau_{1}, \tau_{2}<n_{t+1}$.

Now we construct the desired surface $Y$ (the image surface of $f$ ) by cutting $X$ in a suitable way along rays over $d_{1}, d_{2}, \ldots, d_{N}$. In what follows, we take $0 \leq t \leq r-1$ and $0 \leq \tau<n_{t+1}$. So then, for each such pair $(t, \tau)$, we cut the surface $X$ along a single ray over $d_{\beta(t, \tau)}$, starting from the branch point of $X$ over $w_{\beta(t, \tau)}$.

We now suppose that $p_{n}>p_{k}+1$; since otherwise $p_{k}=p_{n-1}=p_{n}-1$, and this case is covered in Theorem 5 above. Then for each pair $(t, \tau)$ we cut the surface $X$ along $\left(p_{n}-p_{k}-1\right)$ rays over $d_{\beta(t, \tau)}$ not starting from the branch point of $X$ over $w_{\beta(t, \tau)}$.

This gives a simply connected smooth, analytic covering $S$ of $\mathbf{C}$ with the following properties. The surface $S$ covers every point of $\mathbf{C} \backslash\left(\bigcup_{j=1}^{N} d_{j}\right)$ exactly $p_{n}$ times, every point of $\bigcup_{j=1}^{N} \operatorname{Int} d_{j}$ exactly $p_{k}$-times, and every point $w_{\beta(t, \tau)}$ exactly $p_{t+1}$ times. Let $f: \mathbf{B} \rightarrow \mathbf{C}$ be an analytic function whose image surface is $S ; f$ has the valence sequence $p_{n}, p_{n-1}, \ldots, p_{1}$, as desired.

We have now to tackle the case that some of the $n_{j}$ 's vanish. We then have to cut $S$ over additional rays in $\mathbf{C}$ that are mutually disjoint with each other and with the rays $d_{j}$, in such a way that the remaining valencies $p_{j}$ in the sequence $\left\{p_{j}\right\}_{1}^{n}$ are also attained. If $Y$ denotes the resulting surface, then clearly any function $f: \mathbf{B} \rightarrow \mathbf{C}$ with image surface $Y$ satisfies the conclusion of Theorem 6 . This concludes the proof.

Two special cases of Theorems 5 and 6 merit attention:

Corollary 8.1. Every sequence $p_{1}<p_{2}<\cdots<p_{n}$ of non-negative integers that contains two consecutive integers is the valence sequence for a function $f$ which is a polynomial of degree $p_{n}$ of a univalent function.

Corollary 8.2. Every sequence $p_{1}<p_{2}<\cdots<p_{n}$ of non-negative integers that contains two distinct entries $p_{i}$ and $p_{j}$, both less than $p_{n}$, such that $\left|p_{i}-p_{j}\right|$ divides $p_{n}-1$ is the valence sequence of a function $f$ which is a polynomial of degree $p_{n}$ of a univalent function.

Remark. After this paper was submitted for publication, the authors became aware of the independent work of T. Carne, M. Ortel and W. Smith [Bull. London Math. Soc. 19, 1987, 438-442] which contains a weaker form of Theorem 1 (where no exceptional points are allowed) whose proof involves similar ideas.

Acknowledgement. The second author gratefully thanks King Fahd University of Petroleum and Minerals for its support and the Open University for its hospitality during the writing of this paper. Both authors thank Professor K. Stephenson for helpful discussions, particularly in relation to Section 3, and the referee for the careful way he read the manuscript and for a number of valuable suggestions. 


\section{References}

[1] Ahlfors, L.V., and L. SARIo: Riemann surfaces. - Princeton University Press, Princeton, N.J., 1960.

[2] Brannan, D.A., and W.E. Kirwan: Some covering theorems for analytic functions. - J. London Math. Soc. (2) 19, 1979, 93-101.

[3] Burckel, R.B.: An introduction to classical complex analysis, Vol. 1. - Academic Press, New York, 1979.

[4] DugundJI, J.: Topology. - Allyn and Bacon, Inc., Boston, Mass., 1966.

[5] KRA, I.: Automorphic forms and Kleinian groups. - W.A. Benjamin Inc., Reading, Mass., 1972.

[6] Lyzzaik, A., and D. Styer: A covering surface conjecture of Brannan and Kirwan. Bull. London Math. Soc. 14, 1982, 39-42.

[7] Newman, M.H.A.: Elements of the topology of plane sets of points. - Cambridge University Press, Cambridge, 1961.

[8] Ortel, M., and W. SMITH: A covering theorem for continuous locally univalent maps of the plane. - Bull. London Math. Soc. 18, 1986, 359-363.

[9] Pólya, G., and G. Szegö: Problems and theorems in analysis, Vol. 1. - Springer-Verlag, Berlin, 1972.

[10] T. Radó: Zur Theorie der mehrdeutigen konformen Abbildungen. - Acta Litt. ac. Scient. Univ. Hung. (Szeged) 1, 1922/23, 55-64.

[11] Rudin, W.: Real and complex analysis. - McGraw-Hill, New York, 1966.

[12] SPANIER, E.H.: Algebraic topology. - McGraw-Hill, New York, 1966.

[13] Springer, G.: Introduction to Riemann surfaces. - Addison-Wesley, Reading, Mass., 1957.

[14] Srebro, U.: Deficiencies of immersions. - Pacific J. Math. 113, 1984, 493-496.

[15] SRebro, U.: Covering theorems for meromorphic functions. - J. d'Analyse Math. 44, $1984 / 85,235-250$.

[16] Srebro, U., and B. WAJnRYB: Covering theorems for Riemann surfaces. - J. d'Analyse Math. 46, 1986, 283-303.

[17] Srebro, U., and B. Wajnryb: Covering theorems for open surfaces. - Proceedings of the 1985 Georgia Topology Conference: Geometry and topology. Marcel Dekker Inc., Amsterdam, 1987, 265-275.

[18] Titchmarsh, E.C.: Theory of functions. - Oxford University Press, Oxford, 1939.

Open University

Faculty of Mathematics

Milton Keynes MK7 6AA

England

Received 18 April 1986
King Fahd University of Petroleum and Minerals

Department of Mathematical Sciences

Dhahran

Saudi Arabia 